\title{
Effects of temperature and anisotropy on quadrupole absorption in Borrmann spectroscopy
}

\author{
M. Tolkiehn, ${ }^{1, *}$ T. Laurus, ${ }^{1}$ and S. P. Collins ${ }^{2}$ \\ ${ }^{1}$ Deutsches Elektronensynchrotron DESY, FS-DO, Notkestraße 85, D-22607 Hamburg, Germany \\ ${ }^{2}$ Diamond Light Source Limited, Diamond House, Harwell Science and Innovation Campus, Didcot OX11 ODE, United Kingdom
}

(Received 24 October 2011; published 1 December 2011)

\begin{abstract}
We address two important aspects of Borrmann spectroscopy: the dependence of quadrupole enhancement on electronic anisotropy and temperature. A simple framework for describing these effects within the established formalism of dynamical x-ray diffraction theory is presented. This provides a firm basis for Borrmann spectroscopy - a technique for investigating the electronic orbitals that are inaccessible within the electric dipole approximation.
\end{abstract}

DOI: 10.1103/PhysRevB.84.241101

PACS number(s): 61.05.cp, 78.70.Dm

The identification of quadrupole peaks in X-ray absorption spectra is of fundamental importance since they involve transitions to electronic orbitals that play a key role in the electronic properties of the materials, such as the $3 d$ electrons in transition metals. Moreover, these peaks, which tend to appear in the "pre-edge" region of the absorption spectrum, are extremely sensitive to the local environment of the ion. In this Rapid Communication we present a method based on the Borrmann effect ${ }^{1}$ for enhancing these peaks, facilitating their identification as quadrupole peaks and permitting their detailed investigation.

The Borrmann effect describes an increase in transparency to $\mathrm{x}$-ray beams at certain Laue reflections. While the reduction in dipole absorption, caused by the standing-wave field in the crystal, was discovered and described theoretically ${ }^{2}$ half a century ago, it has only been discovered recently ${ }^{3}$ that quadrupole absorption ${ }^{4}$ is enhanced dramatically in the Borrmann effect. For certain reflections the low electric field intensity at the atoms leads to a low dipole absorption. At the same time the high field gradient results in an enhanced quadrupole absorption. Therefore, the intensity of the diffracted beam depends strongly on the quadrupole absorption cross section of the atoms at the nodes of the standing-wave field.

We will describe a theoretical model of the quadrupole enhancement, including a detailed treatment of temperature dependence and anisotropy. This will be demonstrated for the Gd $L_{3}$ edge in gadolinium gallium garnet (GGG) and the Ti $K$ edge in strontium titanate (STO).

Photoelectric absorption, which is the main absorption mechanism for $\mathrm{x}$ rays with energies below $10 \mathrm{keV}$, is caused by excitation of electrons from initial states $\Psi_{i}$ to final states $\Psi_{f}$. Using time-dependent perturbation theory the transition rates $w_{i f}$ can be calculated by considering the x-ray wave field as a perturbation: ${ }^{5}$

$$
w_{i f} \propto|\underbrace{\boldsymbol{E}(0) \cdot\left\langle\Psi_{f}, e \boldsymbol{x} \Psi_{i}\right\rangle}_{E 1}+\underbrace{\left.\sum_{j, l} \frac{\partial E_{l}}{\partial x_{j}}\right|_{x=0}\left\langle\Psi_{f}, e x_{j} x_{l} \Psi_{i}\right\rangle}_{E 2}|^{2},
$$

where $e$ is the elementary charge and $\boldsymbol{E}$ is the electrical field. The total absorption cross section is then proportional to the sum of all transition rates $w_{\text {if }}$ of excitations that fulfill energy conservation. The two terms represent the electric dipole
$(E 1)$ and electric quadrupole (E2) transitions. For $\mathrm{x}$ rays the magnetic terms are extremely weak and have been omitted.

In the Borrmann effect, the electric field $\boldsymbol{E}$ is given by the interference between diffracted and forward diffracted waves. For a Laue reflection there are two pairs of diffracted and forward diffracted waves, which differ both in phase and absorption coefficient. They correspond to points on the two branches of the dispersion surface for $\mathrm{x}$ rays in periodic media. For thick crystals one of these pairs (the $\beta$ branch) can be neglected because its absorption coefficient is usually very high and it cannot be detected at the exit surface. However, the other pair (the $\alpha$ branch) usually has a low absorption coefficient. The wave field of the $\alpha$ branch is given by the dynamical theory of $x$-ray diffraction ${ }^{2,6,7}$ as

$$
\boldsymbol{E}(\boldsymbol{x})=\boldsymbol{E}_{0} e^{i \boldsymbol{K}_{0} \cdot \boldsymbol{x}}+\boldsymbol{E}_{\boldsymbol{H}} e^{i\left(\boldsymbol{K}_{0}+\boldsymbol{H}\right) \cdot \boldsymbol{x}},
$$

with the amplitude of each wave given by

$$
\boldsymbol{E}_{0}=\left(1-\frac{\eta}{\sqrt{\eta^{2}+1}}\right) \frac{\boldsymbol{E}_{i}}{2}, \quad \boldsymbol{E}_{\boldsymbol{H}}=-\beta \frac{1}{\sqrt{\eta^{2}+1}} \frac{\boldsymbol{E}_{i}}{2},
$$

with $\eta=\left[b \Delta \theta \sin 2 \theta_{B}+\frac{1}{2} \Gamma F_{0}(1-b)\right]\left(\Gamma|P| \sqrt{|b|} \sqrt{F_{H} F_{\bar{H}}}\right)^{-1}$, $\beta=\frac{|P| \sqrt{|b|}}{P} \frac{\sqrt{F_{H} F_{\bar{H}}}}{F_{\bar{H}}}, \Gamma=4 \pi r_{e} /\left(k^{2} V\right)$, where $r_{e}$ is the Thomson scattering length, $k$ is the wave vector of the $\mathrm{x}$ rays in vacuum, $V$ is the volume of the unit cell, $\boldsymbol{E}_{i}$ is the amplitude of the incident wave, $\boldsymbol{H}$ the reciprocal lattice vector of the observed Laue reflection, $F_{H}$ the corresponding structure factor, $b$ the asymmetry factor, $P$ the polarization factor, $\theta_{B}$ the Bragg angle, and $\Delta \theta$ the deviation of the incident wave vector from the Bragg condition. Inserting (2) into (1) gives

$$
\begin{gathered}
w_{i f, E 1}(\boldsymbol{x}) \propto\left|\sum_{l}\left(E_{0 l}+E_{\boldsymbol{H} l} e^{i \boldsymbol{H} \cdot \boldsymbol{x}}\right) D_{l}\right|^{2}, \\
w_{i f, E 2}(\boldsymbol{x}) \propto\left|\sum_{j, l}\left(E_{0 l} K_{0 j}+E_{\boldsymbol{H} l} K_{\boldsymbol{H} j} e^{i \boldsymbol{H} \cdot \boldsymbol{x}}\right) Q_{j l}\right|^{2}
\end{gathered}
$$

for the dipole and quadrupole transition rates, where the abbreviations $D_{l}=\left\langle\Psi_{f}, e x_{l} \Psi_{i}\right\rangle$ and $Q_{j l}=\left\langle\Psi_{f}, e x_{j} x_{l} \Psi_{i}\right\rangle$ are introduced. Mixed dipole-quadrupole terms are neglected as they vanish due to inversion symmetry. ${ }^{8}$ For $\sigma$ polarization a coordinate system can be chosen such that $\boldsymbol{H}$ is parallel to the $z$ axis and the electric field is parallel to the $y$ axis. Then the 
transition rates only depend on the matrix elements $D_{2}, Q_{12}$, and $Q_{32}$. For $E 1$ the transition rate is then proportional to the intensity of the interference field of both waves:

$$
w_{i f, E 1}(\boldsymbol{x}) \propto\left|D_{2}\right|^{2}\left[\left|E_{0}\right|^{2}+\left|E_{H}\right|^{2}+2 \operatorname{Re}\left(\overline{E_{0}} E_{H} e^{i \boldsymbol{H} \cdot \boldsymbol{x}}\right)\right] .
$$

This well known fact is the basis for the x-ray standing-wave method. ${ }^{9}$ For E2, the transition rate is more complicated and also depends on the components of the wave vector that is parallel and perpendicular to the lattice planes $k_{\|}$and $k_{\perp}$. After adding up all of the initial and final states, the transition rate is given by

$w_{E 2}(\boldsymbol{x}) \propto k_{\|}^{2} Q^{+}\left|E_{0}+E_{H} e^{i \boldsymbol{H} \cdot \boldsymbol{x}}\right|^{2}+k_{\perp}^{2} Q^{-}\left|E_{0}-E_{H} e^{i \boldsymbol{H} \cdot \boldsymbol{x}}\right|^{2}$,

with

$$
Q^{+}:=\sum_{i, f}\left|Q_{12}\right|^{2} \quad \text { and } \quad Q^{-}:=\sum_{i, f}\left|Q_{32}\right|^{2} .
$$

Note that in the derivation of (6) the cross terms (e.g., $Q_{12} \overline{Q_{32}}$ ) cancel out during the summation over all states. ${ }^{10}$ Using $k_{\perp}=$ $k \sin \theta_{B}$ and $k_{\|}=k \cos \theta_{B}$ the transition rate can be written as

$$
w_{E 2}(\boldsymbol{x}) \propto \sigma\left(\left|E_{0}\right|^{2}+\left|E_{H}\right|^{2}+2 \Xi \operatorname{Re}\left(\overline{E_{0}} E_{H} e^{i \boldsymbol{H} \cdot \boldsymbol{x}}\right)\right),
$$

with

$$
\begin{gathered}
\sigma:=k^{2}\left(Q^{+} \cos ^{2} \theta_{B}+Q^{-} \sin ^{2} \theta_{B}\right), \\
\Xi:=\frac{Q^{+} \cos ^{2} \theta_{B}-Q^{-} \sin ^{2} \theta_{B}}{Q^{+} \cos ^{2} \theta_{B}+Q^{-} \sin ^{2} \theta_{B}} .
\end{gathered}
$$

This result is similar to the dipole absorption (5) except for the dimensionless parameter $-1<\Xi<1$ in the interference term. If the sums in (7) extend over all orbitals in the corresponding subshells, then $Q^{+}=Q^{-}=: Q$ due to symmetry. In this case the parameter $\sigma$ and $\Xi$ have the following values:

$\sigma=\left\{\begin{array}{lll}D & \text { for } & E 1, \\ Q k^{2} & \text { for } & E 2,\end{array}, \quad \Xi=\left\{\begin{array}{llll}1 & \text { for } & E 1, & \sigma \text { pol. } \\ \cos 2 \theta_{B} & \text { for } & E 2, & \sigma \text { pol. }, \\ \cos 2 \theta_{B} & \text { for } & E 1, & \pi \text { pol. }, \\ \cos 4 \theta_{B} & \text { for } & E 2, & \pi \text { pol. }\end{array}\right.\right.$

$D$ is defined in analogy to $Q$ as $D:=\sum_{i, f}\left|D_{2}\right|^{2}=f^{\prime \prime}$, where $f$ is the atomic form factor of the corresponding atom. The calculation of $w_{E 1}$ and $w_{E 2}$ for $\pi$ polarization is not presented here but can be done similarly.

The angular dependence of the absorption can be calculated by inserting (3) into (5) and (8) and evaluating this for all atoms in the unit cell. For a symmetric Laue reflection and $\sigma$ polarization the dipole absorption is

$$
\mu_{E 1}(\eta)=\mu_{0}\left(1-\frac{\operatorname{Im}\left[\left(\sqrt{\eta^{2}+1}+\eta\right) \sqrt{F_{H} F_{\bar{H}}}\right]}{\operatorname{Im} F_{0}}\right),
$$

where $\mu_{0}:=k \Gamma \operatorname{Im} F_{0}$. At the $\operatorname{Bragg}$ maximum $(\eta=0)$, this results in

$$
\mu_{E 1}(0)=\mu_{0}(1-\epsilon) \quad \text { with } \quad \epsilon:=\frac{\operatorname{Im} F_{H} F_{\bar{H}}}{2 \operatorname{Im} F_{0} \operatorname{Re} \sqrt{F_{H} F_{\bar{H}}}} .
$$

When all atoms are located on lattice planes, $\epsilon=1$ and $\mu_{E 1}$ vanishes. This is known as the Borrmann effect.
The quadrupole absorption can be calculated similarly, however, in the most general case the result cannot be expressed in terms of structure factors $F_{H}$ and $F_{\bar{H}}$. In the special case of a crystal with only one sort of atom in the unit cell one obtains

$$
\mu_{E 2}(\eta)=\mu_{0} \frac{Q}{f^{\prime \prime}}\left(1-\Xi \frac{\operatorname{Im}\left[\left(\sqrt{\eta^{2}+1}+\eta\right) \sqrt{F_{H} F_{\bar{H}}}\right]}{\operatorname{Im} F_{0}}\right)
$$

and at the Bragg maximum

$$
\mu_{E 2}(0)=\mu_{0} \frac{Q}{f^{\prime \prime}}(1-\Xi \epsilon) .
$$

In the general case the prefactor is more complicated, but the quadrupole absorption is proportional to $1-\Xi \epsilon$. While dipole absorption is reduced to zero in the Borrmann effect, the quadrupole absorption will be enhanced if $\Xi<0$. For an isotropic environment $\Xi=\cos 2 \theta_{B}$ and an enhancement occurs for $\theta_{B}>\pi / 4$.

Now one can introduce thermal vibrations by including a Debye-Waller factor $e^{-W(T)}$ in the structure factors $F_{H}$ and $F_{\bar{H}}$. This leads to a temperature dependence of the parameter $\epsilon(T)=\epsilon(0) e^{-W(T)}$. For the Borrmann effect, where $\epsilon(0)=$ 1 , the temperature dependence of $E 1$ and $E 2$ absorption is given by

$$
\begin{gathered}
\mu_{E 1}(T)=\mu_{0}\left(1-e^{-W(T)}\right), \\
\mu_{E 2}(T)=\mu_{0, E 2}\left(1-\Xi e^{-W(T)}\right) .
\end{gathered}
$$

A first-order Taylor approximation of the Debye-Waller factor gives

$$
\mu_{E 1}(T)=\mu_{0} W(T), \quad \mu_{E 2}(T)=\mu_{0, E 2}(1-\Xi+\Xi W(T)) .
$$

The dipole absorption will increase with temperature and the behavior of the quadrupole absorption depends on the parameter $\Xi$. If the approximation $W(T) \approx B T$ can be used, ${ }^{11}$ a linear increase with temperature is expected for the dipole absorption. On the other hand, if $B$ is small, the quadrupole absorption will stay constant.

The experiments presented here have been carried out at beamline BM-28 (XMaS) at ESRF using a closed-cycle cryostat mounted on a six-circle diffractometer, operated in vertical scattering mode. ${ }^{12}$ The diffractometer was used to maintain a constant momentum transfer during energy scans. The beam size at the sample was $0.5 \times 0.5 \mathrm{~mm}^{2}$ and the vertical divergence was $0.2 \mathrm{mrad}$.

For the first experimental test of our model we investigated the temperature dependence of the $\mathrm{Gd} L_{3}$ absorption edge in GGG. The first observation of quadrupole enhancement was reported in this material, ${ }^{3}$ but measurements were carried out at only two temperatures and no quantitative interpretation was proposed. In order to test our theoretical model, we measured the intensity of the (008) reflection of a 500- $\mu$ m-thick GGG crystal plate with (100)-oriented surfaces at different energies and different temperatures. The spectra, presented in Fig. 1, show two absorption maxima labeled with $\mathrm{A}$ and $\mathrm{B}$. The total absorption increases with temperature due to lattice vibrations. A precise temperature dependence of each absorption peak can be obtained by fitting the spectra to Voigt profiles. The result is shown in Fig. 2. 


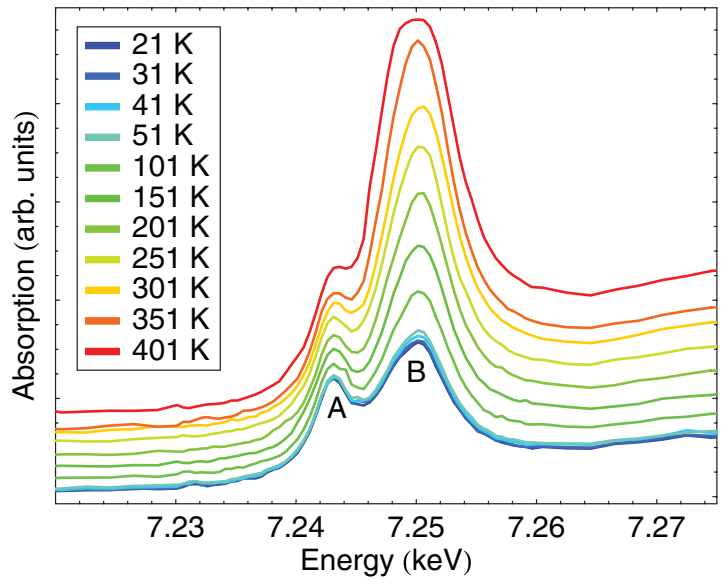

FIG. 1. (Color) Comparison of Borrmann spectra of the GGG (008) reflection measured at different temperatures.

For peak A a very good agreement with an almost constant linear fit can be observed. The deviation at $401 \mathrm{~K}$ is probably caused by the lower counting statistics of the corresponding spectrum. It can be also seen in Fig. 1 that the shape of the curve for $401 \mathrm{~K}$ differs slightly from all the other curves. The constant behavior of peak $A$ is consistent with the assumption that it corresponds to a $2 p \rightarrow 4 f$ quadrupole (E2) transition. Peak B, which has a dipolar nature, shows a linear increase with temperature in the range from 100 to $300 \mathrm{~K}$. The slight deviation from this linear behavior at high temperatures can be attributed to the lower counting statistics at these temperatures. At lower temperatures a saturation effect is observed, which can be attributed to static defects and zero-point lattice vibrations. These observations are in good agreement with our theoretical model and previous observations, which have shown that the ratio $\mu_{E 2} / \mu_{E 1}$ increases at lower temperatures. 3,13

In the second experiment a 500- $\mu$ m-thick, (100)-oriented, 0.13 wt $\%$ iron-doped STO crystal plate was studied. Two different symmetric Laue reflections were measured to demonstrate the dependence on crystal orientation. This effect is caused by the crystal-field splitting. In the octahedral environment the $\mathrm{Ti} 3 d$ orbitals split into $t_{2 g}$ and $e_{g}$ levels,

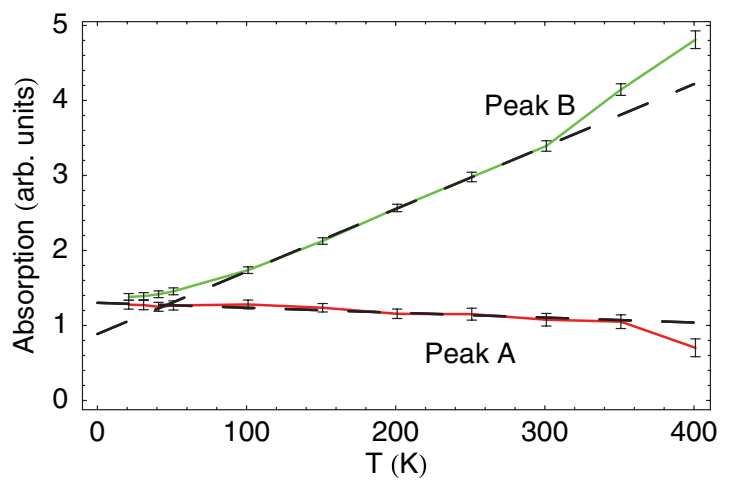

FIG. 2. (Color online) The red (lower) line shows the temperature dependence of the absorption feature at $7.243 \mathrm{keV}$ (peak A), and the green (upper) line corresponds to the feature at $7.250 \mathrm{keV}$ (peak B). In addition, a linear fit (dashed black line) is shown for both curves-for peak B only the range between 100 and $300 \mathrm{~K}$ was used for this fit.

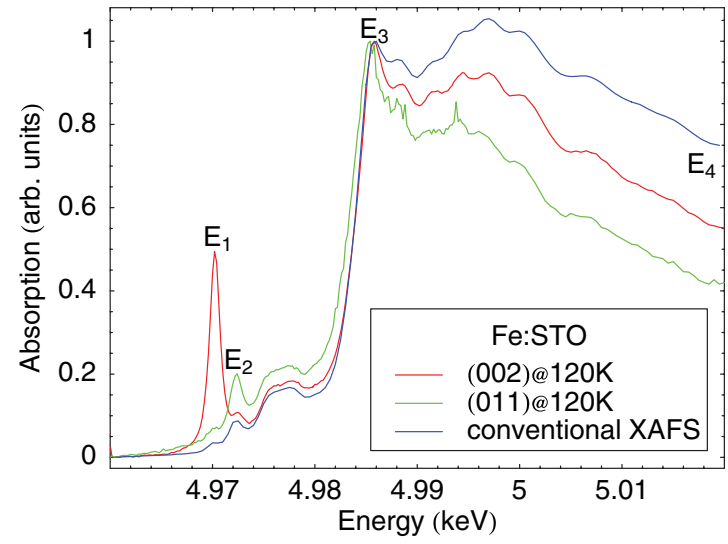

FIG. 3. (Color) Comparison of STO Borrmann spectra measured at different reflections and a conventional XAFS signal, which was measured at beamline A1 at DESY. All curves are normalized to the maximum at $E_{3}$.

which must be taken into account in Eq. (7). In this situation the assumption $Q^{+}=Q^{-}$is no longer valid. We measured the intensities of the (011) and (002) Laue reflections at different temperatures and energies around the Ti $K$ edge. Figure 3 shows the Borrmann spectra at $120 \mathrm{~K}$, just above the cubicto-tetragonal phase transition of STO,${ }^{14}$ in comparison with a conventional x-ray absorption fine structure (XAFS) spectrum. The Borrmann spectra show two strong absorption peaks at energies $E_{1}$ and $E_{2}$, which are not resolved or are very weak in the XAFS curve. These peaks correspond to the $1 s \rightarrow t_{2 g}$ and $1 s \rightarrow e_{g}$ quadrupole transitions, respectively. ${ }^{15}$ The peak

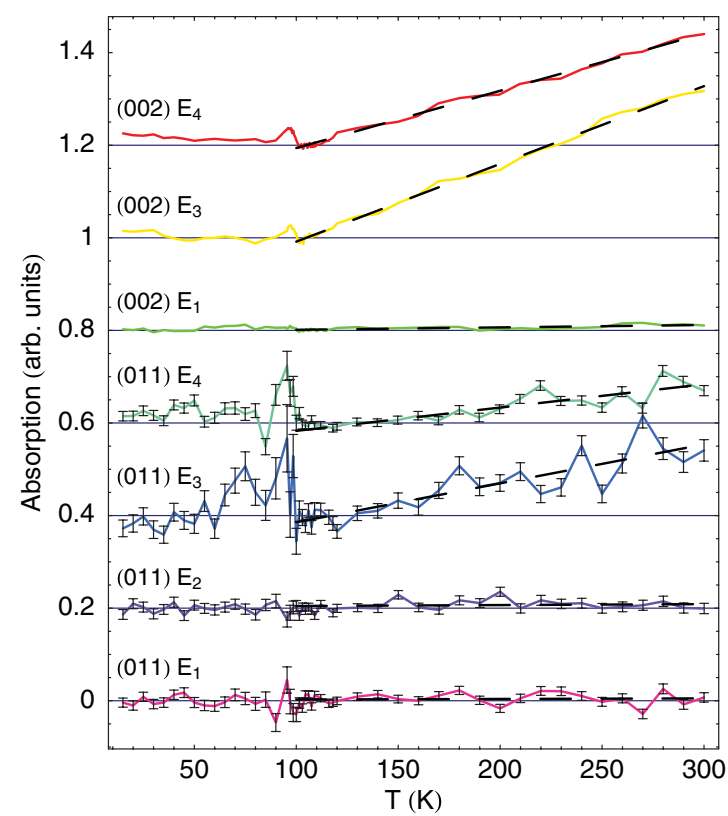

FIG. 4. (Color online) The solid lines show the temperature dependence of the normalized absorption at STO (002) and (011) reflections at the energies $E_{1}=4.97 \mathrm{keV}, E_{2}=4.9722 \mathrm{keV}, E_{3}=$ $4.986 \mathrm{keV}$, and $E_{4}=5.02 \mathrm{keV}$. In addition, a linear fit (dashed black line) is shown for all curves at temperatures above the phase transition. For clarity, the curves are shifted vertically by a multiple of 0.2 . For the (002) reflection (upper three curves) no error bars are shown, because they would be smaller than the line thickness. 
at $E_{1}$ is only observed at the (002) reflection, while the peak at $E_{2}$ is much stronger at the (011) reflection. The dipolar absorption features between $E_{2}$ and $E_{3}$, which correspond to $1 s \rightarrow 4 p$ transitions, are almost identical in all curves.

The difference between the (011) and (002) reflections can be explained by calculating $Q^{+}, Q^{-}$, and $\Xi$ using hydrogenlike wave functions using (7) and (10), taking into account the direction of $\boldsymbol{H}$ and the azimuthal orientation of the sample. For the $(011)$ reflection and $e_{g}$ orbitals as final states one obtains $\Xi=-1$, which leads to the highest possible quadrupole enhancement. For $t_{2 g}$ orbitals as final states $\Xi=1$, which means that quadrupole absorption is suppressed. This is also observed in the experiment. For the (002) reflection, a value of $\Xi \approx 0.18$ is obtained for $t_{2 g}$ final states, which means the transition is slightly suppressed, but still enhanced relative to dipole absorption. For $e_{g}$ final states both $Q^{+}$and $Q^{-}$ are zero, thus the transition is not allowed. The small peak visible in the experimental data at $E_{2}$ could be due to a small dipolar contribution at the same energy. ${ }^{15}$ Although $\Xi>-1$ for (002) at $E_{1}$, the peak is more pronounced than the peak at $E_{2}$ for $(011)$. The (011) reflection is not a perfect Borrmann case $(\epsilon<1)$ because of oxygen atoms located between the diffraction planes, for which the dipole absorption is not suppressed. The enhancement of the quadrupole peaks allowed a precise determination of the crystal-field splitting, determined to be $2.1 \pm 0.2 \mathrm{eV}$.

The temperature dependence of the absorption at four different energies corresponding to the quadrupole resonances ( $E_{1}$ and $E_{2}$ ), the main absorption maximum $\left(E_{3}\right)$, and an energy above the Ti $K$ edge $\left(E_{4}\right)$ is shown in Fig. 4, together with a linear fit for temperatures above the phase transition. A linear increase is obvious at $E_{3}$ and $E_{4}$ for both reflections above the phase transition, while the quadrupole absorption stays constant. This is in good agreement with the theory. At the phase transition an increase of dipole absorption is observed, which is absent or much smaller for the quadrupole peaks. This can be attributed to increased disorder at the phase transition, but cannot be completely explained with our current model.

The quality of the crystals used for this experiment was nearly perfect. The half-width of the measured Bragg reflections was comparable with the beam divergence. Such a high quality is necessary in order to obtain the best results, however, Borrmann spectroscopy can also be applied to mosaic crystals.

Our results show that the resonant Borrmann effect is a powerful tool for investigating the nature of pre-edge peaks in X-ray absorption near edge structure (XANES) spectra. Since quadrupole peaks are enhanced in the Borrmann spectra, this method is better than other methods, which rely on comparing weak peaks in conventional XANES spectra at different angles. ${ }^{16}$ Our technique is very promising for the investigation of $3 d$ states at transition-metal $K$ edges and $4 f$ states at rare-earth $L$ edges, both of which play an important role in electronic and magnetic properties. The simple expressions for quadrupole enhancement, derived from dynamical diffraction theory, have been shown to provide a sound basis for interpreting the measured temperature dependence and anisotropy.

We thank the XMaS staff for support during our beamtimes at ESRF and E. Welter for support at beamline A1 at DESY.

\footnotetext{
*Tolkiehn@gmail.com

${ }^{1}$ G. Borrmann, Physikalische Zeitschrift 42, 157 (1941).

${ }^{2}$ M. von Laue, Röntgenstrahlinterferenzen, 3rd ed. (Akademische Verlagsgesellschaft, Frankfurt am Main, 1960).

${ }^{3}$ R. F. Pettifer, S. P. Collins, and D. Laundy, Nature (London) 454, 196 (2008).

${ }^{4}$ H. Wagenfeld, Phys. Rev. 144, 216 (1966).

${ }^{5}$ F. Schwabl, Quantum Mechanics, 4th ed. (Springer, Berlin, 2007).

${ }^{6}$ A. Authier, Dynamical Theory of X-Ray Diffraction, 2nd ed. (Oxford University Press, Oxford, UK, 2001).

${ }^{7}$ B. W. Batterman and H. Cole, Rev. Mod. Phys. 36, 681 (1964).

${ }^{8}$ M. Blume, in Resonant Anomalous X-ray Scattering, edited by G. Materlik, C. J. Sparks, and K. Fischer (North-Holland, Amsterdam, 1994), p. 495.
}

${ }^{9}$ J. Zegenhagen, Surf. Sci. Rep. 18, 202 (1993).

${ }^{10}$ The details of this calculation will be published elsewhere.

${ }^{11}$ P. Debye, Ann. Phys. 348, 49 (1913).

${ }^{12}$ S. D. Brown, L. Bouchenoire, D. Bowyer, J. Kervin, D. Laundy, M. J. Longfield, D. Mannix, D. F. Paul, A. Stunault, P. Thompson, M. J. Cooper, C. A. Lucas, and W. G. Stirling, J. Synchrotron Radiat. 8, 1172 (2001).

${ }^{13}$ S. P. Collins, M. Tolkiehn, R. Pettifer, and D. Laundy, J. Phys. Conf. Ser. 190, 012045 (2009).

${ }^{14}$ G. Shirane and Y. Yamada, Phys. Rev. 177, 858 (1969).

${ }^{15}$ T. Yamamoto, T. Mizoguchi, and I. Tanaka, Phys. Rev. B 71, 245113 (2005).

${ }^{16}$ S. Bocharov, T. Kirchner, G. Dräger, O. Šipr, and A. Šimnek, Phys. Rev. B 63, 045104 (2001). 